\title{
CARACTERIZACIÓN DE LA EROSIÓN EN ÁREAS ACARCAVADAS DE LA FM. TUDELA (BARDENAS REALES, NAVARRA)
}

\author{
G. DESIR y C. MARÍN \\ Departamento de Ciencias de la Tierra. Facultad de Ciencias \\ Universidad de Zaragoza, 50.009 Zaragoza \\ correo electrónico de contacto: gdesir@unizar.es
}

\begin{abstract}
RESUMEN. En Bardenas Reales, los procesos de erosión se deben fundamentalmente a la acción hídrica y en menor medida actúa la erosión eólica. Los factores que primordialmente controlan la erosión hídrica son el clima, la litología y la geomorfología del terreno. Además de la tasa de la pérdida de sedimentos en suspensión, existen otras formas de exportación de material, mediante coladas de barro y armored mud balls. La presencia de materiales arcillosos con elevadas pendientes y altos índices de dispersión, junto con el clima, condicionan la presencia de un tipo u otro de erosión. En época invernal se desarrollan coladas de barro mientras que en etapas estivales las tormentas de alta intensidad provoca incisión del material. A su vez, las armored mud balls pueden coexistir con ambas formas.
\end{abstract}

ABSTRACT. At Bardenas Reales, erosion processes are due to hydric action and wind erosion. Climate, lithology and geomorphology are the main factors that control water erosion. Besides sediment yield by water, other forms exist to export material: mudslides and armoured mud balls. Clay material at high slopes and high dispersion index together with climate determine the presence of one type of erosion or another. On winter times, mudslides are developed while on summer high intensity storms provoke incision. Meanwhile armoured mud balls could coexist with both of them.

Palabras clave: badlands, gully, coladas de barro, bolas armadas de arcilla, polvo eólico.

Key words: badlands, gully, mud slide, mud armored balls, Aeolian dust.

Enviado el 15 de septiembre de 2008 Aceptado el 20 de diciembre de 2008 


\section{Introducción}

La erosión del suelo constituye la forma más importante de degradación del mismo y el principal factor en relación con la desertificación (López Bermúdez, 2002). Los problemas relacionados con el suelo se ven agravados además por otros factores como la deforestación, el sobrepastoreo y cambios en los usos del terreno (López Bermúdez, 2002). En el año 1991 (PNUMA, 1991) se señalaba que la erosión hídrica es responsable del $48 \%$ de la degradación de las tierras áridas, mientras que la erosión eólica es responsable de un $39 \%$.

Los principales factores que controlan la erosión hídrica son: a) el clima, puesto que condiciona la intensidad, cantidad y duración de las precipitaciones (erosividad de la lluvia); b) la litología, que condiciona la resistencia de un material a ser erosionado (erodibilidad del material); y c) la geomorfología del terreno, puesto que en función de la pendiente del terreno la erosión será mayor o menor. Estos factores, entre otros, fueron analizados por Wischmeier y Smith (1978) para enunciar la Ecuación Universal de Pérdida de Suelo (USLE).

El estudio de los procesos de erosión y su magnitud está tomando gran importancia en los últimos tiempos para su uso en prácticas de conservación agrícolas, fundamentalmente en zonas en donde la erosión es grave o severa. De forma general, se considera que la máxima pérdida anual tolerable de suelo está en $10 \mathrm{Tm} / \mathrm{Ha} / \mathrm{año}$ (Mitchell y Bubenzer, 1980; Mintegui et al., 1993). En cambio, para zonas donde la erosión es severa este límite se establece en $2 \mathrm{Tm} / \mathrm{Ha} / \mathrm{año} \mathrm{(Hudson,} \mathrm{1971)} \mathrm{y} \mathrm{en} 5 \mathrm{Tm} / \mathrm{Ha} /$ año para zonas, como la mediterránea, donde el suelo presenta un escaso desarrollo (Smith y Stamey, 1965). Según la Comunidad Europea, España y Portugal son los países europeos del arco mediterráneo que presentan mayor riesgo de erosión (CORINE, 1992). En el caso de España, el 53\% del territorio está afectado por altas tasas de pérdidas de suelo (López Bermúdez, 2002). Según el Ministerio de Agricultura español (ICONA, 198090) la erosión hídrica media en España es de $27 \mathrm{Tm} / \mathrm{Ha} / \mathrm{año}$, un valor mucho más alto que la media considerada como aceptable. En Bardenas Reales la erosión alcanza valores medios de 32,03 Tm/Ha/año en materiales terciarios y 77,21 Tm/Ha/año en materiales holocenos menos litificados (Desir y Marín, 2007).

Tan importante o más que el estudio cuantitativo de la erosión de una zona determinada, es la identificación y comprensión de los procesos específicos que tienen lugar y que controlan la dinámica erosiva de la misma. Especialmente en estudios encaminados a la protección y recuperación de zonas ya degradadas o donde la erosión antrópica está acelerada o es irreversible. La singularidad y belleza del Parque Natural de las Bardenas Reales radica precisamente en la espectacularidad de sus zonas acarcavadas y en las dimensiones que alcanzan los barrancos erosivos en su interior. Para una misma zona en la que el clima (temperatura, precipitación, evapotranspiración), y cobertera vegetal son similares, los procesos de erosión actuantes pueden ser diferentes dando como resultado morfologías distintas. La consecuencia de esta diversidad morfológica y de mecanismos actuantes hay que buscarla en las propiedades físico-químicas del material (Campbell, 1989). 
En las Bardenas Reales, los gullies aparecen preferentemente desarrollados sobre los materiales limo-arcillosos de edad holocena, mostrando una gran extensión. Las principales características de esta área son la casi total ausencia de relieves elevados y la presencia de numerosos gullies con flancos afectados por procesos de piping y de incisión. Dentro de la depresión erosiva de las Bardenas Reales el paisaje de las acumulaciones recientes cuaternarias se caracteriza por una red de valles de fondo plano incidido por una profunda red de barrancos y un extenso modelado en cárcavas (Gracia, 1985; del Valle y del Val, 1990; del Valle et al., 1991; Leránoz, 1993).

Una de las principales características de la depresión erosiva es la casi total ausencia de relieves importantes en el interior de la depresión y la presencia de numerosos gullies en los que la evolución de las paredes está controlada por procesos de piping y de incisión (Leranoz, 1993). Los gullies principales o de mayor tamaño tienen forma de $\mathrm{U}$ y profundidades que pueden superar $\operatorname{los} 2 \mathrm{~m}$. Los gullies presentan una red dendrítica de alta sinosuidad debido a lo somero del relieve y el principal factor de desarrollo es el piping. La evolución del paisaje en esta área está claramente controlada por la litología y la climatología. Como consecuencia de ello se trata de un modelado muy dinámico y cambiante debido principalmente a los procesos de erosión.

\section{2. Área de estudio}

La zona de estudio, el Parque Natural de Bardenas Reales, se encuentra situada al sureste de la Comunidad Foral de Navarra (Fig. 1A) ocupando 41.845 hectáreas en el sector centro-occidental del valle del Ebro. Comprende una zona de morfología elongada, siendo su eje máximo en dirección N-S de $45 \mathrm{~km}$ y de $24 \mathrm{~km}$ en dirección E-W (Murelaga, 2000). Se trata de una depresión erosiva constituida por materiales de diferentes edades. Los márgenes de la cuenca están formados por arcillas terciarias de la Formación Tudela. Son materiales de edad Mioceno Inferior-Medio (Solé Sedo, 1977;

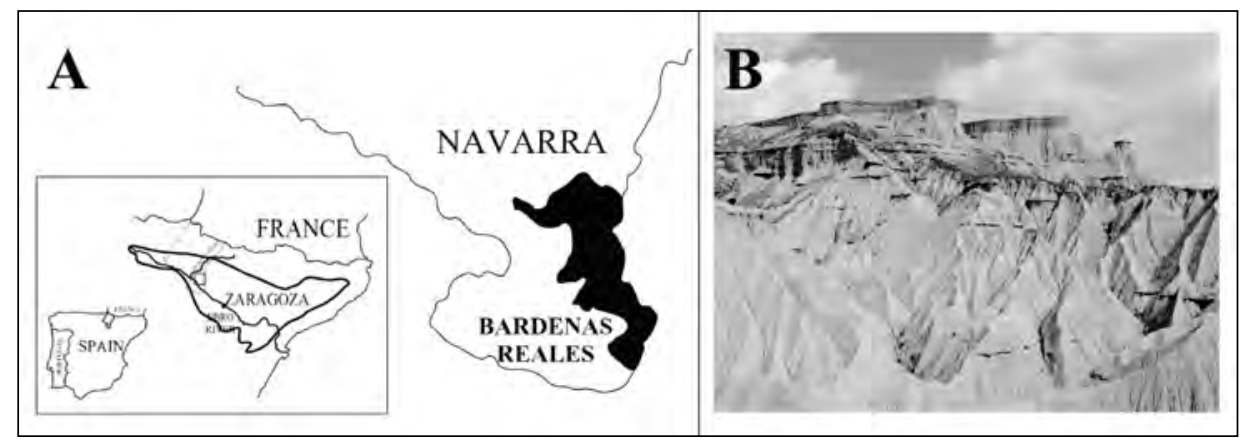

Figura 1. (A) Situación geográfica de Bardenas Reales y (B) vista general de las arcillas terciarias de la Formación Tudela 
Castiella et al., 1978) conformando un paquete de $320 \mathrm{~m}$. de potencia de arcillas versicolores con intercalaciones de calizas, arenas y yesos (Leránoz, 1993; Murelaga, 2000). La zona central de la depresión erosiva se conoce con el nombre de Bardena Blanca, ya que, salvo por la presencia de matorral bajo (ontinas, sisallas), está totalmente desprovista de vegetación y los materiales presentes, arcillas en su mayor parte, adquieren una característica tonalidad blanquecina debido a las eflorescencias salinas que presentan. Se compone de arcillas y limos holocenos poco litificados cuyo origen es el lavado y meteorización de las arcillas terciarias circundantes de la Formación Tudela (Gutiérrez et al., 1995). Sobre este material holoceno se desarrollan profundos gullies de hasta $10 \mathrm{~km}$ de longitud y $8 \mathrm{~m}$ de profundidad, que pueden movilizar hasta $12 \mathrm{~m}^{3} / \mathrm{año}$ de material al río Ebro (Desir y Marín, 2007).

Climatológicamente, la zona de estudio se sitúa en un ambiente semiárido, con una precipitación media anual de $350 \mathrm{~mm} /$ año mientras que la evapotranspiración potencial media (ETP) es de 793 mm (Marín y Desir, 2006). Una de las características de los climas semiáridos es la variación interanual y estacional en las precipitaciones ya que éstas se reparten de manera irregular a lo largo del año (Fig. 2).

Un máximo pluviométrico se da a finales de primavera y otro a comienzos de otoño. Estas lluvias, de carácter ciclonal, son de poca intensidad por lo que su capacidad erosiva es, a priori, baja. Por el contrario, el resto del año, fundamentalmente en verano, la lluvia se producen en forma de tormenta de alta intensidad y por tanto, fuertemente erosiva. Las temperaturas medias de la zona oscilan entre $10^{\circ} \mathrm{C}$ en el mes más frío y $24^{\circ} \mathrm{C}$ en el más cálido, siendo la temperatura media anual de $13^{\circ} \mathrm{C}$. La altitud media de la zona es de 280 m.s.n.m. en la zona central de la depresión erosiva.

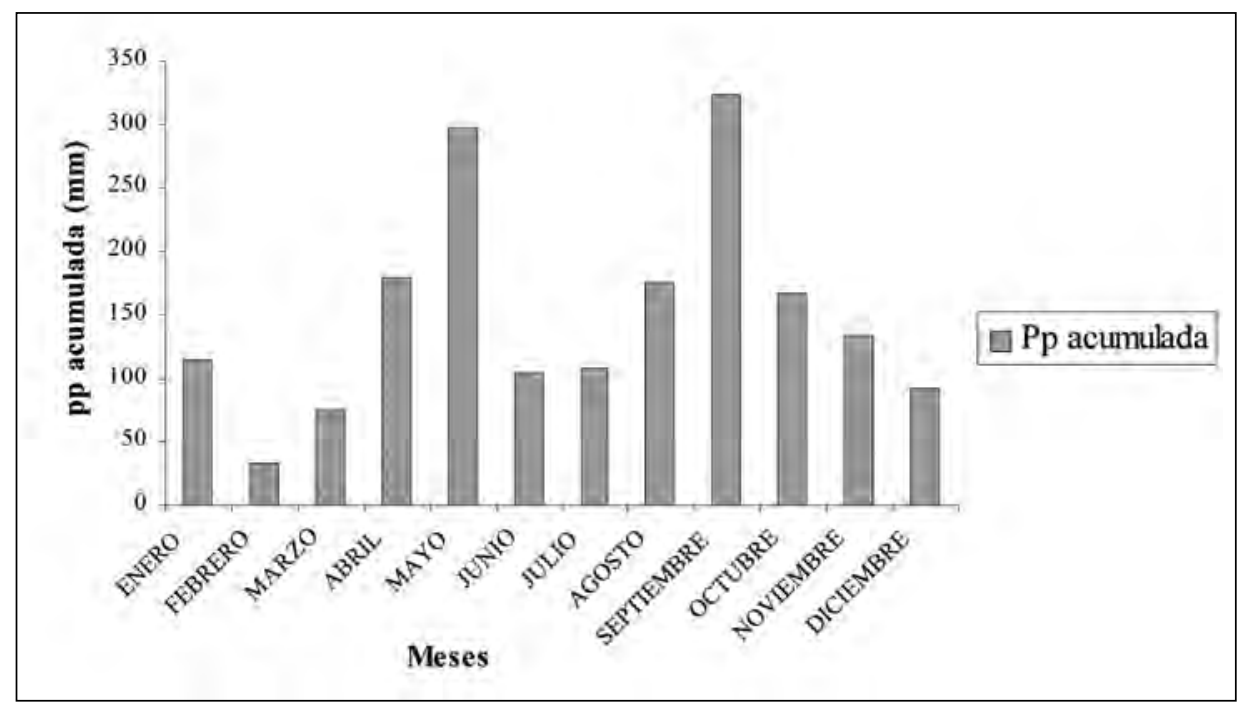

Figura 2. Distribución anual de las precipitaciones en la zona de Bardenas Reales 
Geomorfológicamente, la alternancia de materiales con diferente repuesta a la erosión (arcillas con calizas, areniscas o yeso) en la que predominan claramente los materiales blandos (arcillas y limos) y la disposición horizontal, han permitido actuar a la erosión rápida e intensamente dando como resultado una gran depresión erosiva enmarcada por conjunto de relieves tabulares. Sobre las laderas de estos relieves tabulares y en las faldas de los glacis más antiguos se desarrolla un extenso modelado en cárcavas de laderas muy escarpadas e interfluvios escarpados que son disectados por profundos gullies de paredes escarpadas. El modelado desarrollado sobre los materiales cuaternarios se caracteriza por una red de valles de fondo plano que incide en las mismas elaborando un modelado acarcavado de interfluvios alomados, afectado por procesos de piping (Fig. 3).

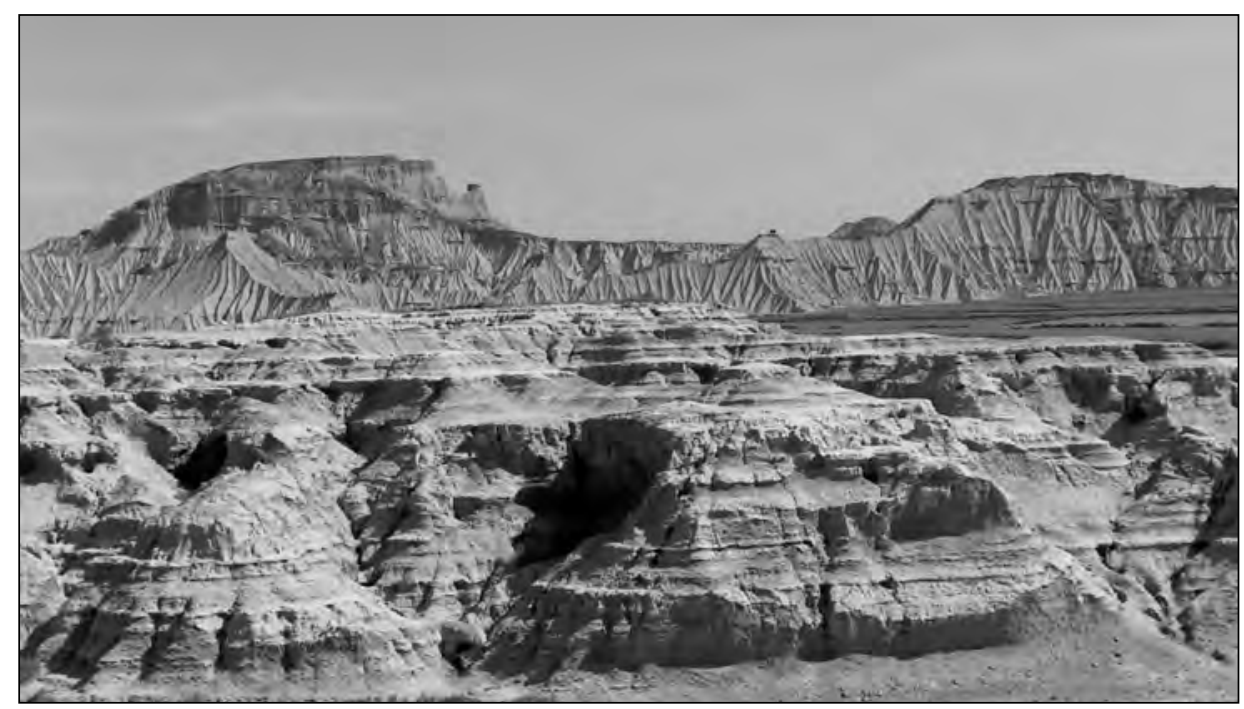

Figura 3. En primer término, modelado desarrollado sobre materiales holocenos y, al fondo, laderas acarcavadas en materiales terciarios

\section{Materiales}

Los materiales terciarios presentes en los márgenes de la depresión erosiva de Bardenas Reales corresponden a la Formación Tudela y se encuentran desprovistos de sus laderas $\left(30^{\circ}-40^{\circ}\right)$, lo que ha favorecido la formación de un espectacular paisaje de de cárcavas (Fig. 1B). Dentro de la Formación Tudela se han diferenciado 5 niveles que se han caracterizado físico-química y mineralógicamente (Tablas 1 y 2). De las propiedades físico-químicas se desprende que se trata de unas arcillas ligeramente dispersivas con estructura masiva, $\mathrm{pH}$ alcalino, alta conductividad eléctrica y ausencia casi total de materia orgánica (Gutiérrez et al., 1995; Desir et al., 2005; Desir y Marín, 2007). 


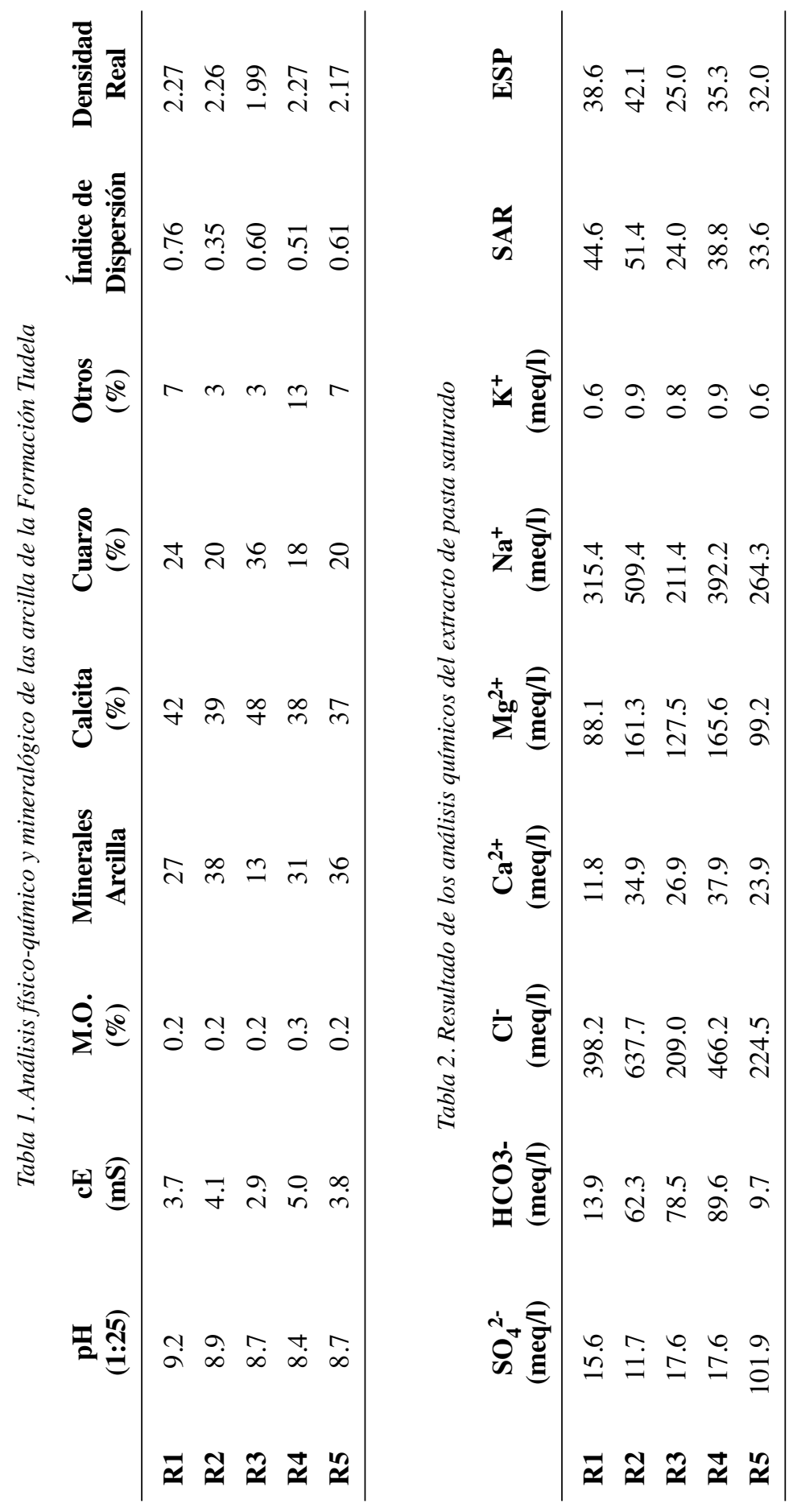


Los análisis mineralógicos realizados con difracción de rayos $\mathrm{X}$ señalan que estos materiales presentan un gran contenido en calcita, con cuarzo y minerales de la arcilla en menor proporción. Mediante el estudio de agregados orientados se estudiaron las especies minerales de arcillas presentes y muestran un alto contenido en illita (82-86\%), un 9-16\% de clorita y trazas de caolinita (Gutiérrez et al., 1995; Desir et al., 2005; Desir y Marín, 2007). La dispersión de las arcillas juega un papel muy importante en los procesos de erosión, ya que es esta dispersión la causante del agrietamiento que genera la pérdida de cohesión de las arcillas acelerando la erosión (Arulanandan y Heinzen, 1977).

El regolito que desarrollan es muy somero, apenas 2-5 cms. Presenta altos valores de SAR y ESP y coeficientes de hinchamiento que pueden alcanzar el 12\% (Gutiérrez et al., 1995). Resultado de ello es el desarrollo de un denso agrietamiento con celdillas poligonales de núcleos abombados, popcorn. Estas morfologías favorecen la percolación y el flujo subsuperficial por lo que se trata de arcillas susceptibles de desarrollar piping. En ausencia de minerales hinchables, como es el presente caso, el hinchamiento y los procesos de piping se deben a altas cantidades de sodio (Jones, 1981; Imeson et al., 1982; Benito et al., 1992; Gutiérrez et al., 1995), como lo demuestran los altos valores de SAR y ESP encontrados en estos materiales. Sin embargo, este está aminorado o no se desarrolla probablemente debido a la alta pendiente que presentan. Estas pendientes evitan que el agua infiltre demasiado en el regolito y se puedan producir pipes.

\section{Procesos de erosión}

La evolución del paisaje en esta área está claramente controlada por la litología y la climatología. La distribución irregular de las precipitaciones, donde alternan máximos pluviométricos con eventos de tormenta con periodos de escasa o nula precipitación, hacen que la estabilidad de estos suelos sea baja y las tasas de erosión altas (Gutiérrez et al., 1995; Sirvent et al., 1997; Marín y Desir, 2004). Por otro lado, las propiedades fisico-químicas de estos suelos condicionan la presencia de procesos de piping y de hinchamiento y consecuentemente la presencia de las diferentes morfologías y mecanismos actuantes (Campbell, 1989).

Las formas de erosión que se desarrollan en Bardenas Reales están principalmente ligadas con la erosión hídrica. Sin embargo, y unido a épocas de sequía prolongada conjugadas con episodios de fuertes vientos se han observado diferentes morfologías generadas por la acción eólica.

\subsection{Acción eólica}

El viento en comparación con el agua resulta un agente erosivo mucho menos intenso, pero en las regiones áridas adquiere una especial importancia. Su principal acción es la de transportar en suspensión las partículas tamaño limo o arcilla, de tal manera que en 
ocasiones se generan tormentas de arena que dificultan la visibilidad. Sin embargo, aunque las formas de erosión eólica son efímeras en la zona de estudio, sí se han observado. Un ejemplo son las dunas obstaculizadas por la vegetación o nebkhas que se formaron en noviembre de 2007 tras una prolongada época sin lluvias (Fig. 5).

Durante la época seca de finales de inverno y principios de primavera cuando la evapotranspiración es baja y no se producen eventos significativos de precipitación la humedad del suelo es fundamentalmente debida a los aportes de las nieblas. Estas son las responsables de la humectación de la costra que posteriormente es desecada durante el día. Los sucesivos ciclos de humectación y secado dan lugar a una intensa meteorización del sustrato. Bajo la capa endurecida de la costra superficial el regolito se encuentra totalmente degradado y disgregado (Fig. 5). Cuando se producen episodios de fuertes vientos estos son capaces de romper la capa protectora debido a la abrasión de las partículas transportadas en suspensión y saltación desencadenando la erosión y exportación del material meteorizado. El resultado es la formación de nubes de polvo, como la originada el 14 de Noviembre de 2007 (Fig. 4) y, el depósito de las partículas eolizadas en las zonas de abrigo, fondos de los regueros o a sotavento de la plantas (Fig. 5). La procedencia de estos materiales como transportados por el viento se evidencia por la alta clasificación granulométrica de este material (Fig. 5).

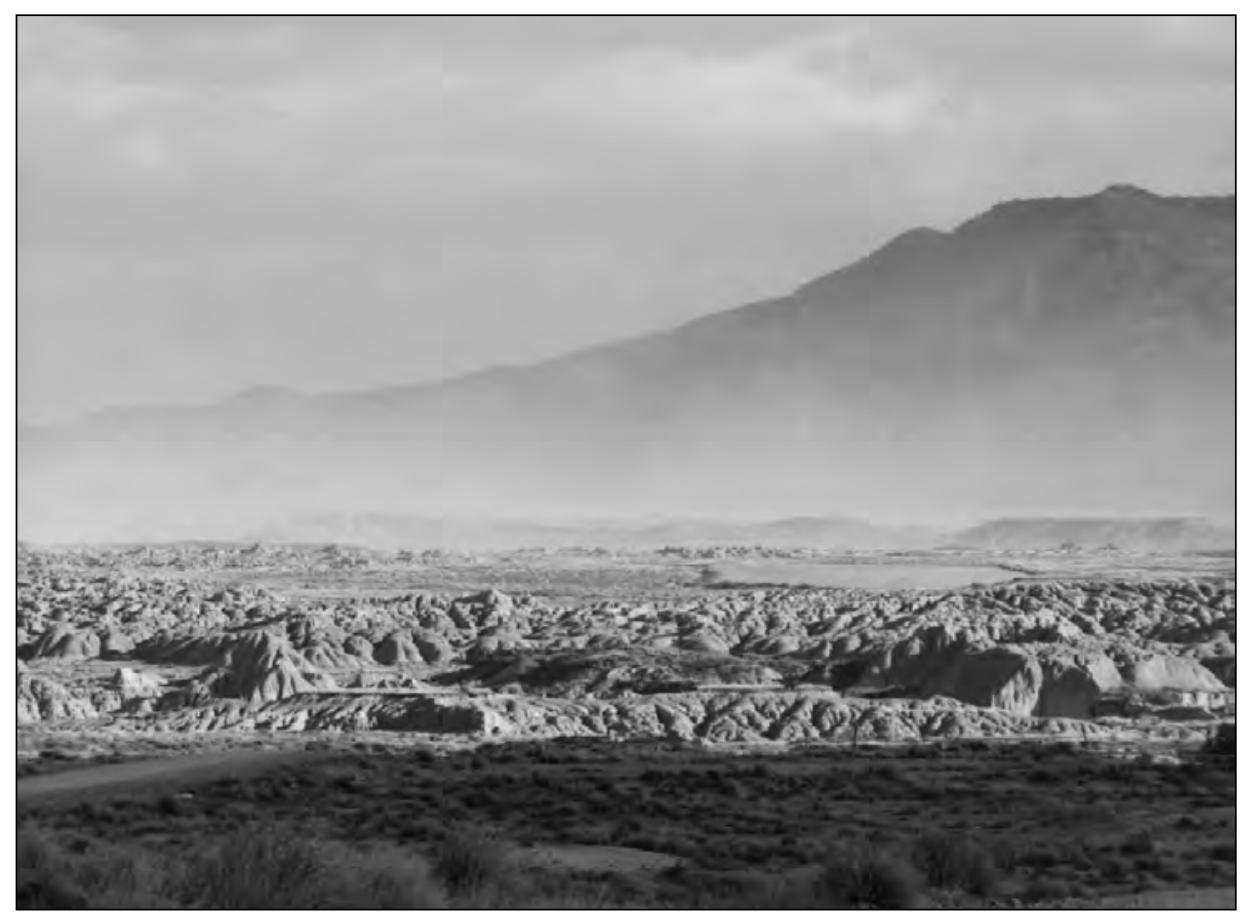

Figura 4. Tormenta de arena generada en noviembre de 2007 en la depresión erosiva de Bardena Blanca 


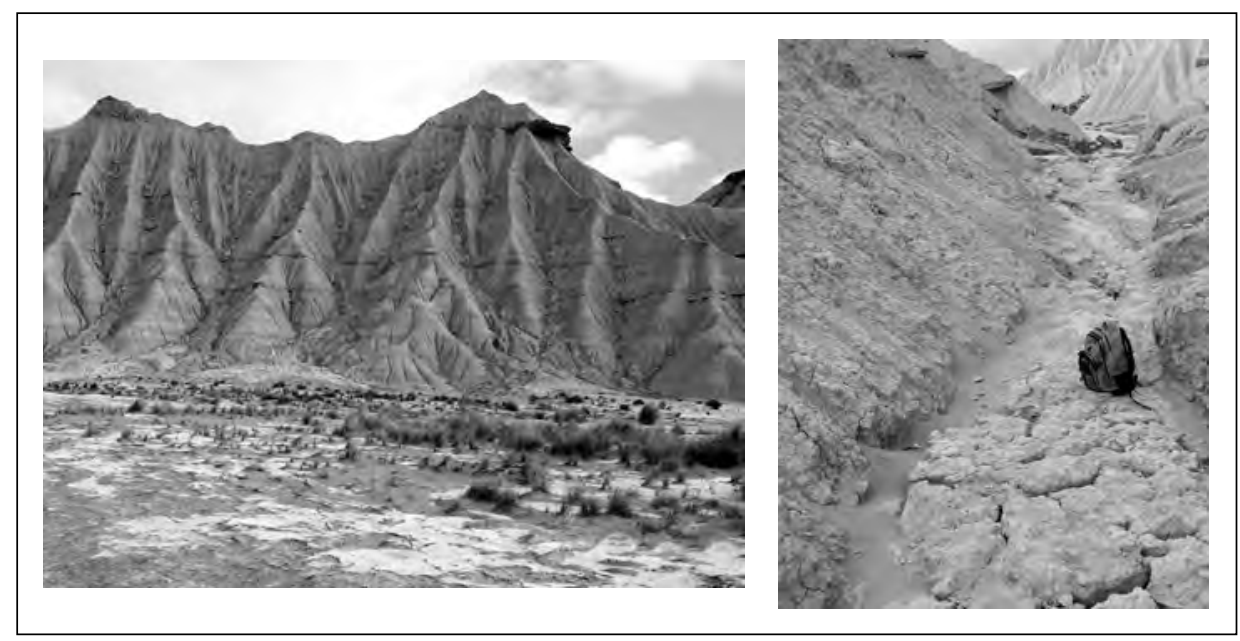

Figura 5. Pequeñas nebkhas (izqda.) generadas por el material disgregado que se acumula en el fondo de rills y gullies (dcha.)

\subsection{Erosión hídrica}

La erosión hídrica es el principal proceso actuante en el medio y el principal responsable del modelado del área. Una de las características principales de esta depresión erosiva es la intensa reguerización que compartimenta pequeñas microcuencas con interfluvios acuchillados y elevadas pendientes a cuyo pie se desarrollan pequeños abanicos. Estos rills son consecuencia directa del arranque y transporte de material por el agua de escorrentía. Sin embargo, la exportación de material en suspensión a través de los rills no es la única forma de erosión hídrica, puesto que en esta zona se han observado otras formas de evacuación por la escorrentía como las bolas armadas de arcilla (armored mud balls).

a.- Pérdida de sedimentos (sediment yield) y Tasa de erosión. El modelado en esta área es muy dinámico y cambiante debido principalmente a los procesos de erosión hídrica. Para conocer el alcance de la misma debido a la acción de la escorrentía superficial se instalaron Parcelas Experimentales para el estudio de la erosión hídrica sobre las arcillas miocenas de la Formación Tudela (Gutiérrez et al., 1995; Sirvent et al. 1997; Marín y Desir, 2004). Mediante esta técnica dinámica se ha podido conocer los valores de pérdida de suelo por erosión hídrica desde el año 1993 en que se instalaron las Parcelas, resultando en una media anual de $32 \mathrm{Tm} / \mathrm{Ha} /$ año (Tabla 3). Las tasas de erosión obtenidas para un periodo de registro de 11 años muestran una gran variabilidad interanual que está contralada por la variabilidad inter e intranual de la precipitación.

Como ya se ha señalado anteriormente la erosión hídrica en estas áreas está controlada por las características del clima y las propiedades del material. De esta manera, Gutiérrez et al., 1995 y Sirvent et al., 1997 señalan la existencia de un doble umbral que controla la producción de sedimentos de las microcuencas desarrolladas sobre las laderas de 
Tabla 3. Valores promedios de las tasas de erosión obtenidas en la Parcela de Erosión BD2 (Bardenas Reales-2) mediante la Parcela de Erosión

\begin{tabular}{|c|c|}
\hline Año & $\begin{array}{c}\text { Bardenas 2 } \\
\text { (datos en Tm/Ha/año) }\end{array}$ \\
\hline 1993 & 89,508 \\
1994 & 55,685 \\
1995 & 25,204 \\
1996 & 35,576 \\
1997 & 21,890 \\
1998 & 6,523 \\
1999 & 32,633 \\
2000 & 41,357 \\
2001 & 1,703 \\
2002 & 38,367 \\
2003 & 15,496 \\
2004 & 20,504 \\
\hline Media & $\mathbf{3 2 , 0 3 7}$ \\
\hline
\end{tabular}

los materiales arcillosos. Existe una relación directa entre la cantidad de precipitación y la escorrentía generada reconociéndose un umbral en torno a los $11 \mathrm{~mm}$, que señala la ausencia de escorrentía por debajo de esta precipitación. La relación entre intensidad máxima y sedimentos es directa para precipitaciones por encima del umbral y por debajo del mismo la producción de sedimentos es casi nula o inexistente (Gutiérrez et al., 1995).

Existe una clara relación entre los distintos eventos de precipitación y la producción de escorrentía y sedimentos (Fig. 6). Se observan en las curvas acumulativas dos claros escalones que van ligados a sucesos de alta magnitud correspondientes a lluvias de carácter convectivo que tuvieron lugar a final de primavera y comienzos de otoño. La producción de sedimentos originada por estos dos eventos es más del doble que la generada a lo largo de todo un año. Esto nos indica que la erosión está controlada fundamentalmente por lluvias de tormenta. Entre los escalones se sitúan etapas de escasa producción de escorrentía y sedimentos, que se localizan durante el verano, finales de otoño, invierno y la mayor parte de la primavera. Las precipitaciones durante estas épocas o no tienen lugar o corresponden a lluvias ciclonales de baja cantidad e intensidad en los que la erosión hídrica es pequeña.

En la base de las cárcavas desarrolladas al pie de los relieves tabulares y de los glacis más elevados se extiende una amplia red de profundos gullies que canalizan la escorrentía y representan la principal vía de exportación de los materiales movilizados. En las Barde- 


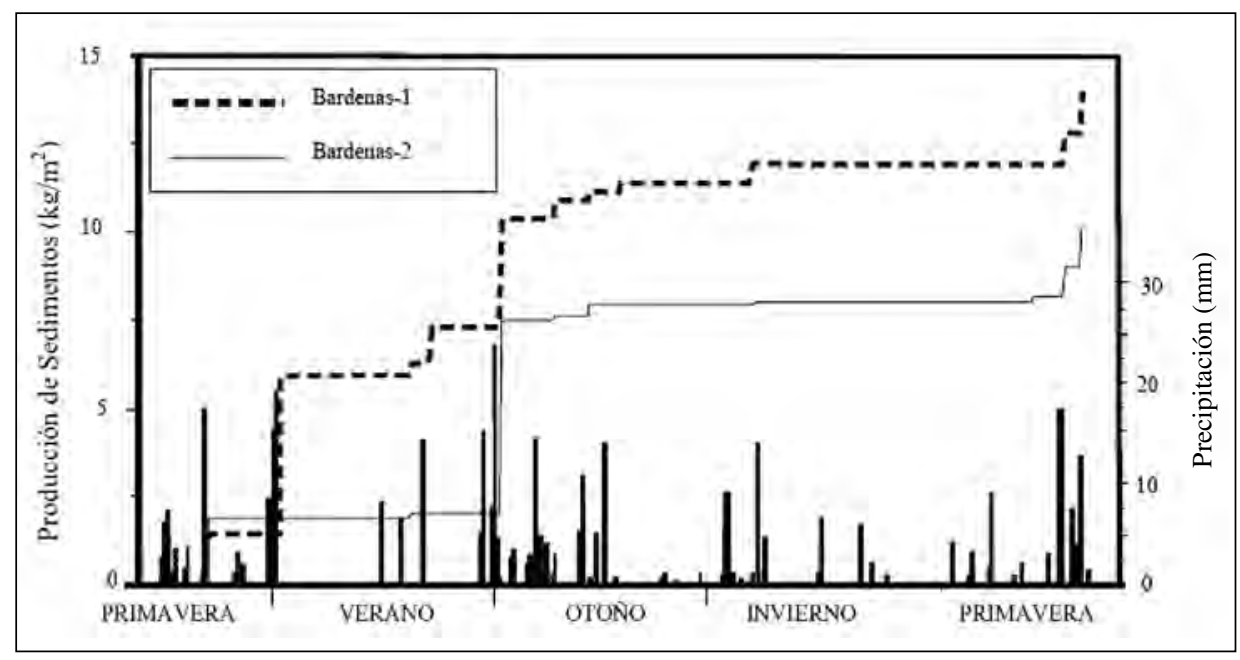

Figura 6. Relación entre la precipitación y producción de sedimentos. Obsérvese como los máximos de lluvia coinciden con escalones en la curva acumulativa de sedimentos

nas Reales, la evolución de los gullies responde a diferentes procesos como retroceso de cabeceras, arroyada, socavación basal y piping. FAO (1978) señala que la evolución de los gullies se produce mediante varios procesos, los cuales pueden tener lugar al mismo tiempo o por separado. Estos son incisión del fondo y las paredes, y la erosión por el efecto cascada en la cabecera, lo que da lugar a un importante retroceso de la cabecera y el cual está sujeto al volumen y velocidad de la escorrentía. En nuestro caso la secuencia evolutiva establecida pasa por un primer estadio erosivo, con predominio de la incisión y de expansión lateral por el colapso de los conductos de piping. A continuación se produce una etapa donde el retroceso de la cabecera es el proceso dominante propiciado por la caída de bloques y vuelcos, que posteriormente en la fase final serán lavados y exportados.

b.- Armored mud balls. Los materiales terciarios presentan además de la pérdida de sedimentos en suspensión por la arroyada, otra forma de exportación y pérdida de suelo, que aunque menor es destacable. Estas formas son las armored mud balls. Se pueden definir como una forma mixta entre la erosión hídrica y los movimientos de masas. En primer lugar porque necesita de la presencia de unos flujos con una condiciones hidrodinámicas determinadas para su formación, redondeamiento y posterior armadura. Y los movimientos de masas porque suplen el material original que posteriormente es retrabajado y modelado en bolas. Las armored mud balls se definen como clastos redondeados con un núcleo de arcilla o arcillo-limoso y un revestimiento o "armazón” de arena, gravilla o gravas.

Las armored mud balls se distribuyen a lo largo de las cuencas siguiendo una ordenación que nos evidencia su origen y proceso de formación. El esquema general de formación comenzaría en las laderas donde se formarían, favorecidas por el desarrollo de grietas, popcorn y otros procesos de meteorización física, pequeños bloques de arcilla 
más o menos angulosos que por gravedad se depositan al pie de las mismas y que son movilizados en los siguientes eventos con escorrentía. En trabajos previos las coladas de barro han sido descritas como fuente de bloques irregulares de arcillas, que por procesos de transporte y erosión posterior se van redondeando y armando dando como resultado armored mud balls (Kluger y Saunders, 1959; Chun et al., 2002). El tamaño y forma final de las bolas está condicionado por el espaciado y profundidad del agrietamiento (Martins et al., 2003).

Posteriormente, y en su evolución a lo largo de la cuenca se observa como en la parte alta de la cuenca donde el gully discurre muy encajado entre paredes muy verticalizadas, la mayoría de bolas son de pequeño tamaño, ligeramente irregulares y con armaduras muy sutiles o inexistentes. En las zonas donde la cuenca se abre y el gully se ensancha y someriza las bolas presentan mayores dimensiones, especialmente aquellas que se encuentran sobre las barras o interfluvios. En las partes distales de la cuenca las bolas son muy numerosas y presentan una distribución al azar solo controlada por los obstáculos y la vegetación que actúa como trampa para las mismas (Fig. 7). En este sector el tamaño es menor que en el tramo intermedio por lo que puede interpretarse que las bolas comienzan a erosionarse hasta llegar a desaparecer en la zona distal.

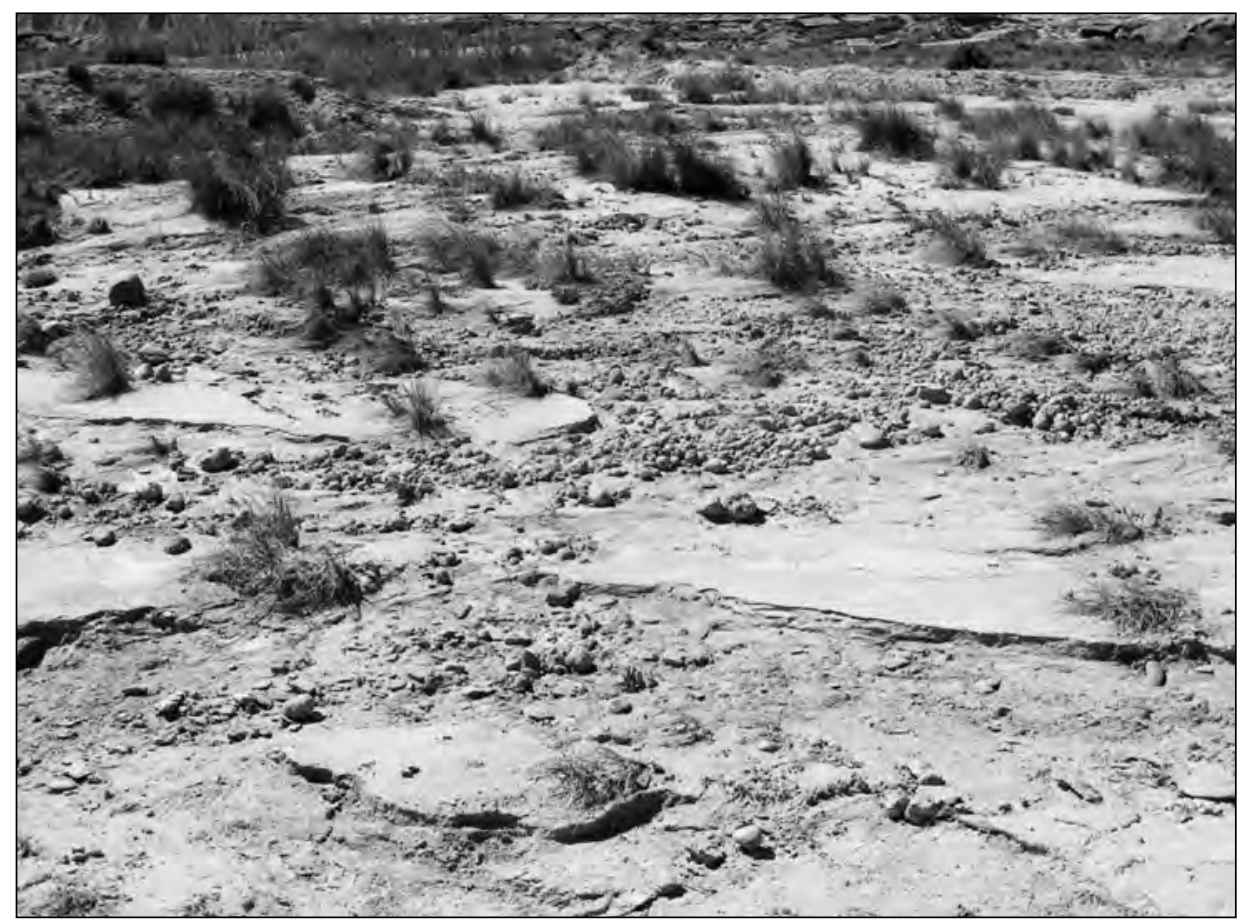

Figura 7. Armored mud balls retenidas por la presencia de vegetación 


\subsection{Movimiento de masas}

Los suelos dispersivos presentan unas características mecánicas y geotécnicas muy particulares. Los materiales arcillosos de la Fm. Tudela se caracterizan por presentar unos elevados contenidos en sodio, elevadas pendientes $\left(30-40^{\circ}\right)$; una densa red de drenaje; índices de plasticidad altos; abundantes grietas de desecación. Todos estos factores junto con prolongados periodos de sequía y una alta variabilidad estacional de las precipitaciones propician que durante las épocas invernales, cuando la evapotranspiración es mínima y el grado de humedad del suelo alcanza su máximo, se generen importantes coladas de barro o mud slides. La formación de estas coladas está controlada por el comportamiento plástico de estas arcillas y su rápida transición al comportamiento fluido (Marín y Desir, 2006).

Las coladas de barro se definen como un movimiento de masas en arcillas, limos o arenas de grano fino que, como consecuencia de haber alcanzado su límite plástico, son capaces de desplazarse deslizando con un movimiento lento y resultan en formas lobuladas o elongadas (Hutchinson y Bhandari, 1971; Brundsen, 1984; Allison y Brundsen, 1990; Brundsen e Ibsen, 1996). Generalmente en las áreas de cárcavas las coladas de barro se canalizan a favor de los regueros preexistentes de modo que éstos pueden quedar enmascarados llegando en algunos casos a desaparecer. Sin embargo, estas no son las únicas zonas donde se desarrollan las coladas, pues también se han identificado sobre las laderas (Fig. 8A), especialmente en aquellas orientadas al norte. Las coladas desarrolladas en Bardenas Reales suelen ser peliculares puesto que el regolito tiene poco espesor.

Por otro lado, la zona donde se produce el depósito del material movilizado tiene forma lobulada y puede estar formada por un único lóbulo o por una superposición de varios (Fig. 8B). Como consecuencia de la agradación en la zona apical, la longitud del canal se va reduciendo puesto que el ápice del lóbulo se encuentra cada vez más cerca de la zona de arranque y consecuentemente también se reduce la pendiente del canal por el que circula. Cuando la lengua deslizada es inactiva y las condiciones climáticas favorables, esta puede llegar a erosionarse e incluso desaparecer volviéndose a formar los regueros preexistentes.

Las variables climáticas son los principales agentes de control en la formación de las coladas de barro. La intensa meteorización sufrida durante el periodo seco junto con la gradual entrada de humedad favorecida por la escasa intensidad de las lluvias y el desarrollo de un agrietamiento profundo han generado profundos perfiles de meteorización de elevada porosidad y escasa cohesión. Estos mantos de alteración son fácilmente movilizados y alcanzan grandes distancias cuando sobrepasan el límite líquido. Cuando las lluvias son de baja intensidad son capaces de infiltrar en el regolito de tal manera que éste adquiere la humedad necesaria para alcanzar el límite plástico y poder deslizar. Por el contrario las lluvias de alta intensidad y baja duración son muy erosivas y eliminan las coladas formadas durante la etapa de lluvias calmas. 


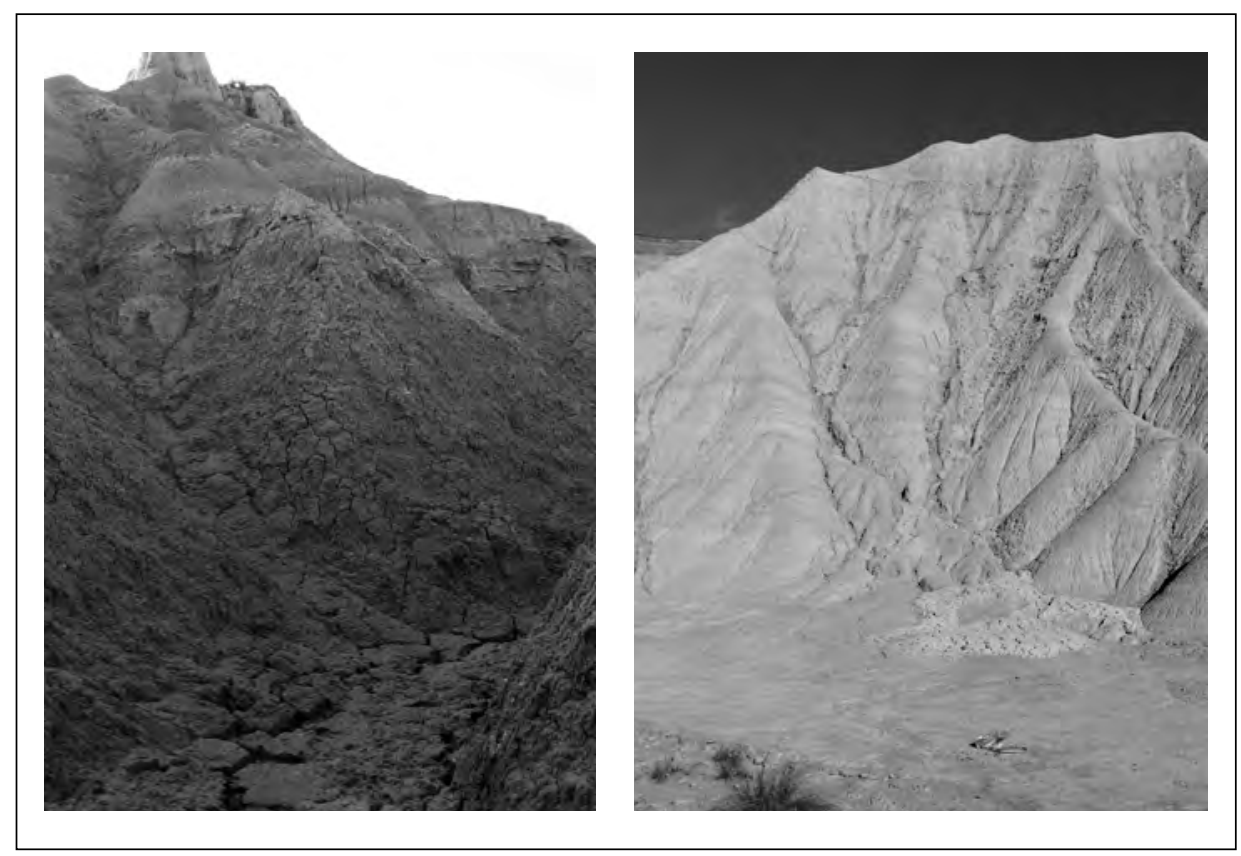

Figura 8. (A). Colada de barro sobre una ladera que desemboca en otra colada en el fondo de un rill.(B). Superposición de varios lóbulos al pie de una ladera

\section{Resultados y discusión}

Las formas de erosión que se desarrollan en Bardenas Reales están principalmente ligadas con la erosión hídrica. Solé Benet (2006) en su análisis de la erosión en España indica que las Bardenas Reales son las parcelas experimentales para el estudio de la erosión en las que se han registrado las tasas de erosión más altas. Estas elevadas tasas de erosión son consecuencia directa de las propiedades físico-químicas del material y de la elevada irregularidad intra- e interanual de las precipitaciones. Son suelos altamente erosionables frente a la lluvia y a la escorrentía consecuencia principalmente del elevado contenido de sodio en la fracción arcillosa, cuyo lavado provoca la destrucción de la estructura. Sin embargo, y unido a épocas de sequía prolongada conjugadas con episodios de fuertes vientos se han observado diferentes morfologías generadas por la acción eólica.

Las formaciones arcillosas terciarias son fácilmente degradadas por procesos de meteorización como el haloclastismo, debido al lavado de sales, humectación y secado, hinchamiento y contracción debido a la presencia de arcillas hinchables y altos contenidos de sodio. Los amplios periodos secos seguidos de eventos pequeños y aislados favorecen el desarrollo de grietas, popcorn. Los ciclos de humectación y secado generan contracciones e hinchamientos de las arcillas y la solubilización o estabilización de las sales da lugar a la meteorización del sustrato generando las característica estructura en pop- 
corn. En las épocas secas el agrietamiento alcanza su máximo facilitando la infiltración mediante la creación de vías de acceso al sustrato de la escorrentía generada. En épocas húmedas, las grietas se cierran debido al hinchamiento del material. Gutiérrez et al., 1995 señalan que el hinchamiento puede llegar a ser del 12\% en ausencia de minerales de la arcilla hinchables.

La producción de sedimentos en las formaciones terciarias de las Bardenas Reales muestran la alternancia de periodos de gran producción de sedimentos, que tienen lugar por la actividad de lluvias de tormenta fundamentalmente durante la primavera y otoño, con otros de escasa pérdida de suelo correspondientes al resto de las estaciones. De los datos obtenidos mediante parcelas experimentales se observa una relación directa entre la precipitación y la producción de sedimentos, reconociéndose en este caso un umbral de precipitación para la generación de escorrentía y la producción de sedimentos. También se ha observado, como existen eventos anómalos de precipitación muy baja y de gran producción de sedimentos que corresponden la generación de coladas de barro (Gutiérrez et $a l ., 1995)$. Estos flujos se desarrollan en épocas de lluvias ciclonales cuando el material de relleno del reguero y de las áreas de interrill alcanza estados de elevada plasticidad.

Las coladas de barro en el área estudiada se producen durante los meses de invierno. En este periodo las lluvias son escasas y de baja intensidad. Con esas características, las precipitaciones son capaces de ir infiltrando poco a poco en el regolito. Esto, unido a la presencia de al menos 25 días al año de heladas y la baja evapotranspiración en los meses de invierno hacen que el sedimento vaya lentamente empapándose en agua y adquiera su límite plástico. En ese momento y si se supera el ángulo de fricción las arcillas son capaces de deslizar y producir las coladas de barro. En general, estas coladas se mantienen hasta las lluvias de primavera ya que entonces las precipitaciones son de carácter tormentoso (corta duración y alta intensidad) (Fig. 2) y desdibujan en la mayoría de los casos las morfologías existentes. Para que las coladas se desarrollen es necesario que el grado de humedad de la ladera se mantenga durante un periodo relativamente largo. En nuestro caso la ausencia de vegetación, la lluvia de poca intensidad durante el periodo frío, el hecho de que la evapotranspiración es menor en ese momento del año y que las laderas sobre las que se desarrollan se encuentren en las zonas de umbría, hacen que se acumule la humedad necesaria para producir las coladas de barro.

Se ha observado que ambos procesos, la formación de mud slides y de armored mud balls, son excluyentes dado que las condiciones de formación son totalmente contrarias. Las armored mud balls necesitan para su formación eventos de alta energía que genere flujos de escorrentía concentrados con unas características hidrodinámicas suficientes para erosionar y modelar las armored mud balls. La forma y anchura del cauce controlan la velocidad de la escorrentía y la altura de la lámina de agua y estas la creación o destrucción de las armored mud balls. La formación de las coladas de barro requiere de unas condiciones de humedad elevadas, estas mismas condiciones pueden provocar la destrucción de las bolas. Dickas y Lunking, (1968) señalan que la destrucción de los agregados se produce por la humectación del núcleo, o por la destrucción del núcleo por la acción de la helada. Sin embargo, está clara la relación existente entre ambas pues las 
armored mud balls se formarían como paso previo las coladas de barro, que posteriormente actuarían como área fuente de las mismas. La formación de las armored mud ballss de mayor tamaño se debería a la meteorización de las coladas de barro, donde el patrón de agrietamiento previo al mud slide controlaría el tamaño de las bolas resultantes.

\section{Conclusiones}

Existe una clara interrelación entre los diferentes procesos de erosión en las formaciones miocenas de las Bardenas Reales. Las principales formas de erosión están ligadas a la actividad hídrica puesto que las condiciones necesarias para que actúe la erosión eólica son muy puntales y rara vez se producen. La presencia de elevadas pendientes desarrolladas sobre materiales arcillosos con elevados índices de dispersión, altos SAR y ESP condiciona junto con las variables climáticas la presencia de un tipo u otro de erosión.

Las morfologías derivadas de la erosión por la arroyada pueden llegar a coexistir en el tiempo. Sin embargo, las condiciones de formación de unos u otras las hace excluyentes. Así, en épocas invernales de escasa evapotranspiración y precipitaciones de baja intensidad es fácil, debido a la elevada pendiente, la formación e coladas de barro. Cuando en épocas estivales se producen eventos de tipo tormenta de eleva intensidad y/o volumen, domina la incisión y tiene lugar la profundización de los regueros. Entre ambas formas coexisten las armored mud balls que pueden tener su origen bien en fragmentos disgregados de las coladas de barro en las épocas secas como de la caída de fragmentos de las laderas, consecuencia de la gravedad y de la intensidad del agrietamiento. $\mathrm{Su}$ preservación temporal depende de la intensidad y volumen de la precipitación durante los máximos pluviométricos y del grado de humedad que puedan alcanzar en las épocas invernales.

\section{Referencias bibliográficas}

Allison, R.J., BRUNDSEn, D. (1990). Some mudslide movements patterns. Earth Surface Processes and Landforms 15: 297-311.

Arulanandan, K., HeinZEN, R.T. (1977). Factors influencing erosion in dispersive clays and methods of identification. Erosion and solid matter transport in inland waters. IAHS, 122: 75-81.

Benito, G., GutiérREz, M., SANCHO, C. (1992). Erosion rates in badlands areas of the central Ebro Basin (NE Spain). Catena 19: 269-286.

Brundsen, D. (1984). Mudslides. En: Slope Instability (Brundsen, D., Prior, D.B., Eds.). John Wiley \& Sons, $620 \mathrm{pp}$. 
BRUndSEN, D., IBSEN, M.L. (1996). Mudslides. En: Landslide Recognition: Identification, movement and causes (Dikau, R., Brundsen, D., Schrott, L. Ibsen, M.L., Eds.). John Wiley \& Sons, 251 pp.

CAMPBELl, I.A. (1989). Badlands and badland gullies. En: Arid Zone Geomorphology (Thomas, D., Ed). Ed. Belhaven Press, 372 pp., London,

Castiella, J., Solé, J., Del Valle, J. (1978). Mapa Geológico de Navarra E. 1:200.000. Cartografía Geológica a partir de la Investigación Geológica de Navarra a escala 1: 25.000. Diputación Foral de Navarra. Ser. Geol. Dir. O.P. Pamplona.

Chun, S.S., ChOE, M.Y., ChOugh, S.K. (2002). Armored mudstone boulders in submarine debris-flow deposits, the Hung-hae formation, Pohang Basin: An evidence for the large-scale slumping of adjacent area of a submarine channel or scar wall. Geosciences Journal, 6 (3): 215-225.

CORINE (1992). Soil Erosion Risk and Important Land Resources in the Southern Regions of the European Community. Commission of the European Communities. EUR 13233, 97 pp., Luxemburg.

Del Valle de LeRsundi, J., Del Val, J. (1990). Procesos de erosión y análisis de sus condicionantes en una región semiárida: la cuenca de Cornialto (Bardenas, Navarra). II Reunión del Cuaternario Ibérico. Madrid.

Del Valle de Lersundi, J., Del Val, J., Donezar, M. Y ArrizabalagA, B. (1991). La erosión en Navarra. Navarra Agraria, 64: 34-53.

DEsIR, G., MARÍn, C. (2007). Factors controlling the erosion rates in a semi-arid zone (Bardenas Reales, NE Spain). Catena 71: 31-40.

Desir, G., Marín, C., Guerrero, J. (2005). Badlands and Talus Flatirons in the Bardenas Reales Region. Field Trip Guide. VI International Conference on Geomorphology. 37 pp. Zaragoza.

DiCKAS, A.B., LUNKING, W. (1968). The origen and destruction of armored mud balls in a fresh water lacustrine environt, Lake Superior, Journal of Sedimentary Petrology, 38: 1366-1484.

FAO (1978). Soil Erosion by water: some measures for its control on cultivated land. FAO Land and Water Development Series 7m, 284 pp.

GRACIA, F.J. (1985). Geomorfología de las Bardenas Orientales. Tesis de Licenciatura. Facultad de Ciencias. Universidad de Zaragoza. 172 pp.

Gutiérrez, M., Sancho, C., Desir, G., Sirvent, J., Benito, G., Calvo, A. (1995). Erosión Hídrica en Terrenos Arcillosos y Yesíferos de la Depresión del Ebro. Ministerio de Agricultura, Pesca y Alimentación. Universidad de Zaragoza. 389 pp. Zaragoza. 
HutCHINSON, J.N., BHANDARI, R. (1971). Undrained loading: a fundamental mechanism of mudflows and other mass movements. Geotechnique, 21: 353-358.

Hudson, N.W. (1971). Conservación del Suelo. Editorial Reverte. 335 pp. Barcelona.

ICONA (1980-1990). Mapas de Estados Erosivos. Cuencas Hidrográficas del Ebro, Guadiana, Guadalquivir, Júcar, Pirineo, Segura, Sur de España, Tajo... ICONA. Ministerio de Agricultura, Pesca y Alimentación. Madrid.

IMESON, A.C., KwAAD, F.J.P.M., Verstraten, J.M. (1982). The relationship of soil physical and chemical properties to the development of badlands in Morocco. In: Badland Geomorphology and Piping (Bryan, R.B., Yair, A., Eds.). GeoBooks. 47-70 pp. Norwich.

JONES, J.A.A. (1981). The nature of soil piping: a review of research. British Geomorphological Research Group Monograph Series 3. Geobooks, 301 pp. Norwich.

KLuger, H.G., SAUnders, J.B. (1959). Occurence of armored mud balls in Trinidad, West Indies. Journal of Geology, 67: 563-565.

LERÁNOZ, B. (1993). Geomorfología y Geología Ambiental de la Ribera de Navarra. Ph.D. Thesis. Faculty of Science. University of Zaragoza.

LóPeZ-Bermúdez, F. (2002). Erosión y desertificación. Heridas de la Tierra. Nívola Ediciones. 200 pp. Madrid.

MARín, C., DESIR, G. (2004). Influencia de las propiedades físico-químicas del regolito en los procesos de erosión. Bardenas Reales (Navarra). En: Riesgos naturales y Antrópicos en Geomorfología (Bienes, R. y Marqués, M.J., Eds.). Sociedad Española de Geomorfología. 543 pp. Madrid.

MARÍN, C., DESIR, G. (2006). Descripción morfológica y origen de las coladas de barro desarrolladas sobre arcillas terciarias en la Depresión de Bardenas Reales (Depresión del Ebro). En: Geomorfología y Territorio. IX Reunión Nacional de Geomorfología (Pérez-Alberti, A. y López Bedoya, J., Eds.), 221-234 pp., Santiago de Compostela.

MARTinS, L.R., TABAJARA, L.L. (2003). Ocorrência de fragmentos de Lama na Praia do Cassino, RS, Brasil. Gravel, 1: 47-53.

Mintegui, J.A., De Simón, E., García RodrígueZ, J.L., Robredo, J.C. (1993). La restauración hidrológico-forestal en las cuencas hidrográficas de la vertiente mediterránea. Junta de Andalucía, 325 pp., Sevilla

Mitchell, J.K., Bubenzer, G.D. (1980). Soil Loss Estimation. In: Kirkby, M.J., Morgan, R.C.P. (Eds.). Soil Loss Estimation. Wiley, pp. 17-62, Chichester. 
Murelaga, X. (2000). Estudio de las faunas de vertebrados del Mioceno Inferior de las Bardenas Reales y áreas colindantes (Cuenca del Ebro, Navarra). Ph.D. Thesis. Faculty of Science. University of Pais Vasco.

Pnuma (1991). A New Assessement of the World Status of Desertification. United Nations. Nairobi.

SMith, R.M., STAMEY, W.L. (1965). Determining the range of tolerable erosion. Soil Science 100: 414-424.

Sirvent, J., Desir, G., GutiÉrRez, M., SANChO, C., Benito, G. (1997). Erosion rates in badlands areas recorded by collectors, erosion pins and profilometer techniques (Ebro basin, NE Spain). Geomorphology, 18: 61-75.

Solé Benet, A. (2006). Spain. En Soil Erosion in Europe (Boardman, J. y Poesen, J., Eds.). Wiley.

Solé Sedo, J. (1977). Mapa Geológico de España E. 1:50.000. Hoja 282, Tudela. IGME. $2^{\text {a }}$ Serie, $1^{\text {a }}$ edición, 18 p., 1 mapa. Madrid.

WischMEIER, W.H., SMITH, D.D. (1978). Predicting Rainfall Erosion Losses. Agricultural Handbook, $\mathrm{n}^{\circ}$ 537, 58 pp. USDA. Washington D.C. 